Methods 32 rabbits were randomly divided into four groups: normal control group (CN group), diabetic group (DM group), diabetic pioglitazone treatment A group (DPG group, $4 \mathrm{mg} /$ day $/ \mathrm{kg}$ ) and diabetic pioglitazone treatment B group (DPI group, $8 \mathrm{mg} / \mathrm{day} / \mathrm{kg}$ ). All rabbits were monitored hemodynamics and recorded aortic systolic and diastolic blood pressure (SBP and DBP) and left ventricle end diastolic pressure (LVEDP) and underwent transthoracic echocardiographic examination. After 8-week treatment, whole-cell patch-clamp technique was used to measure action potential duration (APD) and atrial ionic currents (ICaL and INa). Isolated Langendorff perfused rabbit hearts were used to evaluate atrial electrophysiological parameters. Atrial collagen volume fraction (CVF) was calculated by Sirius-Red (SR) staining for pathological examination. Western-blot was applied to assess atrial protein expression levels of NF- $\mathrm{B}$ p $50 / \mathrm{p} 65$, TGF- $\beta$, HSP70, ERK1/2 and p-ERK1/2.

Results Echocardiographic examination showed that LAD, IVST and PWT of DPG and DPI group were significantly decreased compared with DM group. SBP and DBP of DPI group were significantly lower than DM group $(p<0.05)$. No significant difference of LVEDP was among the four groups $(p>0.05)$. Histopathological examination revealed that left atrialmyocytes disordered accompanied by inflammatory cells infiltration and a large amount of interstitialfibrosis distributed throughout the tissue in DM group. SR staining showed that left atrium collagen volume fraction (LACVF) was significantly increased in DM group $(p<0.01)$. But these pathological abnormal findings were attenuated in DPG and DPI group. Compared with CN group, IACT was prolonged ( $p<0.01)$, AERPD was increased $(p<0.01)$ in DM group. Inducibility of AF in DM group was significantlyhigher than controls ( $6 / 8$ vs $1 / 8$ of animals, $p<0.01)$. Inducibility of $A F$ in DPI group was significantly less than DM group $(2 / 8$ vs $6 / 8$ of animals, $\mathrm{p}<0.01$ ). Prolonged IACT, AERPD, AF episode and enhanced inducibility of AF were attenuated in DPG and DPI group. APD90 andAPD50 of left atrial myocyte were prolonged in DM group $(p<0.05$ vs control), and APD90 rate adaptation was no significant differences ( $p>0.05$ vs control). The densities of INa were reduced and the densities of ICaL were increased in DM group ( $p<0.01$ vs control), which were attenuated in DPG and DPI group. TGF- $\beta$, NF$\kappa B$ p50/p65, HSP70, ERK1/2, p-ERK1/2 were increased significantly in $\mathrm{DM}$ ( $\mathrm{p}<0.05 \mathrm{~s}$. control). Protein expression of ERK1/2 was no significant difference between DPG or DPI group and DM group ( $p>0.05)$, but protein expressions of NF- $\mathrm{BB}$ p50/p65, HSP70, TGF- $\beta$ andpERK1/2 were significantly decreased in DPG and DPI group $(p<0.05)$, but no significant differences between two pioglitazone groups.

Conclusions Pioglitazone inhibited DM-related arrhythmogenic atrial remodelling and vulnerability to AF. Pioglitazone may play important preventions from AF in diabetes. PPAR- $\gamma$ activators could become novel upstream therapy drugs for DM-related AF.

\section{GW23-e1035 PIOGLITAZONE PREVENTS DIABETES-RELATED ATRIAL FIBRILLATION SUSCEPTIBILITY IN ALLOXAN-INDUCED DIABETIC RABBITS}

doi:10.1136/heartjnl-2012-302920a.33

Changle Liu, Huaying Fu, Jian Li, Lijun Cheng, Xinghua Wang, Tong Liu, Guangping Li, Tong Liu. Department of Cardiology, Tianjin Institute of Cardiology, Second Hospital of Tianjin Medical University, Tianjin 300211, China

Objectives In the present study, we investigated underlying mechanisms for the effects caused by pioglitazone, a peroxisomeproliferatoractivated receptor (PPAR)- $\boldsymbol{\gamma}$ activator, on atrial structural remodelling and electrical remodelling in alloxan-induced diabetic rabbits. 